\title{
Análisis bibliométrico sobre heladas en cultivos y su relación con las soluciones generadas de diferentes investigaciones
}

\section{Bibliometric analysis of frost in crops and its relationship with the solutions generated from different investigations}

DOI: 10.46981/sfjhv2n3-007

Received in: April 1st, 2021

Accepted in: May 31th, 2021

\author{
Harold Steven Moreno Vargas \\ Seccional Tunja. \\ E-mail: Harold.moreno@usantoto.edu.co \\ Carlos Alberto Cardona Coy \\ docente facultad de Ingeniería Electrónica, \\ E-mail: carlos.cardona@usantoto.edu.co, \\ Luz Angela Cuellar Rodríguez \\ Docente Facultad de Ingeniería Ambiental. \\ Universidad Santo Tomás, Seccional Tunja \\ E-mail: Dec.ambiental@usantoto.edu.co
}

Estudiante de Ingeniería Ambiental Facultad de Ingeniería Ambiental, Universidad Santo Tomás,

\section{RESUMEN}

Se analizaron un total de 40 artículos de investigación sobre el fenómeno de las heladas, afectaciones que tienen las heladas, sistemas de riego y soluciones y/o estrategias que se pueden encontrar hoy en día para minimizar el daño causado por este fenómeno durante el periodo comprendido entre 2001 a 2021, las bases de datos utilizadas fueron Science Direct y Scopus, de igual forma se encontraron artículos en repositorios de universidades a nivel nacional y asimismo en Google Scholar. En el análisis se incluyen las palabras clave, el año de publicación, enfoque, países, número de citas y número de autores. El número de publicaciones presentó un incremento durante los últimos años. Los resultados indicaron que "frost", "frost problem", "irrigation systems", "frost solutions" fueron los términos en los cuales se hizo más frecuente en los títulos de las publicaciones, mediante el uso de los índices bibliométricos de productividad se encontró que el índice de colaboración más alto fue en los años de 2015 y 2019, durante los años de 2001, 2005, 2007 se presentó el índice Price más alto. La universidad Santo Tomás fue la institución más productiva en cuanto a desarrollo de investigación referentes a los temas, de igual forma a nivel nacional, otras universidades tuvieron un porcentaje de publicaciones con los temas relacionados, lo que indica que a nivel nacional este tema ha venido tomando mucha fuerza con relación a las variaciones climáticas que se presentan hoy en día. Los países con más números de publicaciones encontrados fueron Colombia, Brasil, Estados Unidos. Con relación a los idiomas encontrados por las publicaciones, se encontró un total de $42,5 \%$ en español, un $40 \%$ en inglés y un 17,5\% en portugués.

Palabras clave: Análisis bibliométrico, heladas, problemática sobre heladas, sistemas de riego, soluciones a heladas. 


\section{ABSTRACT}

A total of 40 research articles were analyzed on the phenomenon of frost, effects of frost, irrigation systems and solutions and/or strategies that can be found today to minimize the damage caused by this phenomenon during the period from 2001 to 2021, the databases used were Science Direct and Scopus, Articles were also found in national university repositories and in Google Scholar. The analysis includes keywords, year of publication, focus, countries, number of citations and number of authors. The number of publications has increased in recent years. The results indicated that "frost", "frost problem", "irrigation systems", "frost solutions" were the terms in which it became more frequent in the titles of the publications, using bibliometric productivity indices found that the highest collaboration index was in 2015 and 2019, during the years 2001, 2005, 2007 the highest Price index was presented. The Santo Tomás University was the most productive institution in terms of research development related to the topics, similarly at the national level, other universities had a percentage of publications with related topics, this indicates that at the national level this theme has been gaining a lot of strength in relation to the climatic variations that are presented today. The countries with the greatest number of publications found were Colombia, Brazil, United States. Regarding the languages found in the publications, a total of $42.5 \%$ were found in Spanish, $40 \%$ in English and $17.5 \%$ in Portuguese.

Keywords: Bibliometric analysis, frost, frost problems, irrigation systems, frost solutions.

\section{INTRODUCCIÓN}

Las heladas ocurren cuando la temperatura del aire cae a $0^{\circ} \mathrm{C}$ o menos, llegando a un punto de congelación del agua afectando de manera irreversible los organismos vegetales. Este fenómeno provoca grandes pérdidas en la agricultura de las zonas más altas del país ubicadas por encima de los 2.500 m.s.n.m. como los altiplanos fríos, especialmente el altiplano cundiboyacense y otros (Estacio Rodriguez \& Amp; Ariza Garzón, 2020). Hoy en día, las acciones tomadas por parte de los agricultores cuando se presenta el fenómeno de las heladas estiman demasiados recursos e insumos que presentan un aumento del presupuesto y la inversión que tienen ellos para sus cultivos. De las acciones que tienen los agricultores podemos encontrar la de mantener húmedo el follaje de la flor, otro es el de aplicar suplementos nutritivos sobre la planta donde este método aumenta su resiliencia y capacidad de soportar cambios de temperatura y otra es la de instalar barreras mecánicas que ayudan a disminuir los efectos negativos de los fuertes vientos. Cada acción que se menciona tiene un alto valor y ahí es donde el presupuesto del agricultor se ve afectado (González Botero, 2018). El fenómeno de la helada en Colombia es demasiado frecuente, más de lo que se podría esperar y es debido a esto que es causante de millonarias pérdidas a la agricultura de las tierras altas del país, sobre todo en áreas localizadas a más de 2.500 m.s.n.m. especialmente en los meses secos del año (IDEAM (Instituto de hidrología meteorología y estudios ambientales), 2012).

Los altos costos de producción y la instalación de diferentes sistemas de proyección, el riego, la compra de agroquímicos y demás factores que se tienen que tener en cuenta a la hora de cultivar, dejan 
ver la desventaja que tienen los pequeños productores, ya que las pocas ganancias que ellos reciben de sus cosechas las invierten en pagar terrenos arrendados, mano de obra contratada o créditos bancarios obtenidos, siendo así causa que en más de una oportunidad la cosecha por diferentes razones y una de ellas las heladas, generen únicamente grandes pérdidas económicas. Así mismo las heladas y demás factores que afectan los cultivos pueden llegar acabar con las cosechas parcial o totalmente, se registró que, hasta el mes de febrero del año 2020, más de 67.000 hectáreas de pastos y cultivos que se vieron afectados por las fuertes heladas, llevando a cabo la perdida de alrededor de 43.000 de las 150.000 hectáreas que tiene Boyacá para la producción de alimentos. Por consiguiente, esto indica que la mayoría de las veces los agricultores tienen que invertir los créditos bancarios en la compra de nuevos nutrientes y/o gastos adicionales en la restauración del terreno para salvar la cosecha de plantas que sobreviven a los impactos de las heladas (Estacio Rodriguez \& Amp; Ariza Garzón, 2020).

La heladas siempre han sido tema importante en los agricultores y aún más las estrategias que ellos utilizan para minimizar este impacto y que sus cultivos no se vean tan afectados. Es por esta razón, que el desarrollo de esta investigación fue diseñado con el propósito de conocer totalmente el fenómeno de las heladas, sus afectaciones a los cultivos y demás, de igual manera conocer todo lo relacionado con los sistemas de riego, como están compuesto y por último, las diferentes estrategias que los agricultores a nivel mundial realizan a sus cultivos, todo esto se realizó durante los años 2001 a 2021. Los datos para la realización de la investigación bibliométrica fueron filtrados de diferentes bases de datos como: Web of Science, Science Direct y Scopus, de igual manera se buscó en los diferentes repositorios de las universidades a nivel nacional, asimismo se encontraron varios artículos en Google scholar. Asimismo, dentro de este análisis realizado se tiene en cuanta diferentes aspectos como lo son: los años de publicación, número de autores, número de citas, tipo de documento, los diferentes países donde se realizaban investigaciones en este tema y palabras clave. Los resultados nos proporcionaron información relevante sobre las tendencias de investigaciones que se han venido realizando, las cuales pueden ayudar a otros investigadores a identificar la investigación que se ha realizado sobre el tema principal que es el fenómeno de las heladas y asimismo, las estrategias existentes y los sistemas de riego.

\section{METODOLOGÍA}

Para realizar el análisis bibliométrico de este artículo, se realizó una cadena de búsqueda en la cual se utilizaron diferentes bases de datos como: Science Direct y Scopus, de igual manera se buscó en los diferentes repositorios de las universidades a nivel nacional, asimismo en Google Scholar, donde se encontraron varios artículos. Aparte de esto, se debió tener en cuenta alguno criterios de búsqueda como lo fueron: 
- El filtro por publicación, donde allí se localizaron exclusivamente artículos referentes a las diferentes áreas de investigación.

- El filtro por palabra clave, donde se localizaron los artículos que tuvieses las palabras claves como lo fueron: "heladas", "sistemas de riego", y "soluciones a las heladas".

- El filtro por las temáticas importantes en la investigación, donde se localizaron artículos que abordaran específicamente los temas relacionados a las heladas, las características de las heladas, sistemas de riego, qué criterios se necesitaban para hacer un sistema de riego y las diferentes soluciones que los agricultores le daban a las heladas, a nivel mundial.

Al realizar la revisión de las diferentes bases de datos y repositorios de las universidades, se encontraron un total de 65 artículos, de los cuales 25 tuvieron que ser descartados ya que al inicio de la búsqueda coincidían los términos, pero al revisarlos detalladamente tenían un enfoque diferente, o no tenían nada que ver con la investigación que se está realizando, la búsqueda se realizó con un rango de años entre 2001, a 2021, haciendo uso del idioma inglés, portugués y español.

Con base en la información obtenida en las bases de datos y repositorios de las universidades, se aplicaron las diferentes leyes de la bibliometría mediante el uso de indicadores bibliométricos que dieran cuenta de las características de los diferentes artículos y revistas donde estos se publicaron, asimismo como es el caso de índice de colaboración que es la media ponderada de la actividad y cooperación científica entre varios autores por documento, de igual forma se aplicó el índice de Price, donde este nos muestra la proporción del número de referencias menor a cinco años anteriores, con respecto al total de las referencias.

\section{RESULTADOS Y DISCUSIÓN}

Se analizaron en total 65 artículos publicados con relación a todo lo que tiene que ver con el fenómeno de las heladas, los sistemas de riego, como están compuesto, que tipos se pueden encontrar hoy en día, de igual manera, las soluciones que les dan los agricultores hoy en día a este fenómeno, todo esto se hizo durante los años 2001 a 2021, de los cuales se descartaron un total de 25 artículos, debido a que a la hora de revisarlos detalladamente no tenían ninguna relación con el enfoque buscado para la revisión y/o lo mencionado anteriormente. Dentro de los parámetros analizados se incluyeron: tipo de documento, países y de igual forma, se analizó el número de citas que tenía cada artículo.

Con un total de 40 documentos, donde se encontró que el tipo de documento que predomina es el artículo científico, con el 100\% de los documentos. Se analizaron los datos de los números de citas de cada artículo como lo podemos observar en la tabla 1, encontrando que, de los 40 artículos de investigación revisados, alrededor de 383 es el total de las citas de los artículos encontrados. 
Tabla 1. Total, de investigaciones sobre heladas, sistemas de riego y soluciones a las heladas en un periodo comprendido de 2001 y 2021.

\begin{tabular}{|l|c|c|}
\hline TIPO DE DOCUMENTO & TOTAL ARTÍCULOS & \# DE CITAS TOTALES \\
\hline INVESTIGACIÓN & 40 & 383 \\
\hline
\end{tabular}

Como se muestra en la figura 1, la investigación sobre lo relacionado a las heladas, los sistemas de riego y las estrategias que se encuentran hoy en día para mitigar los daños causados por las heladas, han venido aumentando a lo largo de los años. El número de autores también ha venido aumentando a lo largo de los años finales, esto indica que ha tenido una mayor cooperación al pasar los años.

Figura 1. Número de artículos publicados por año.

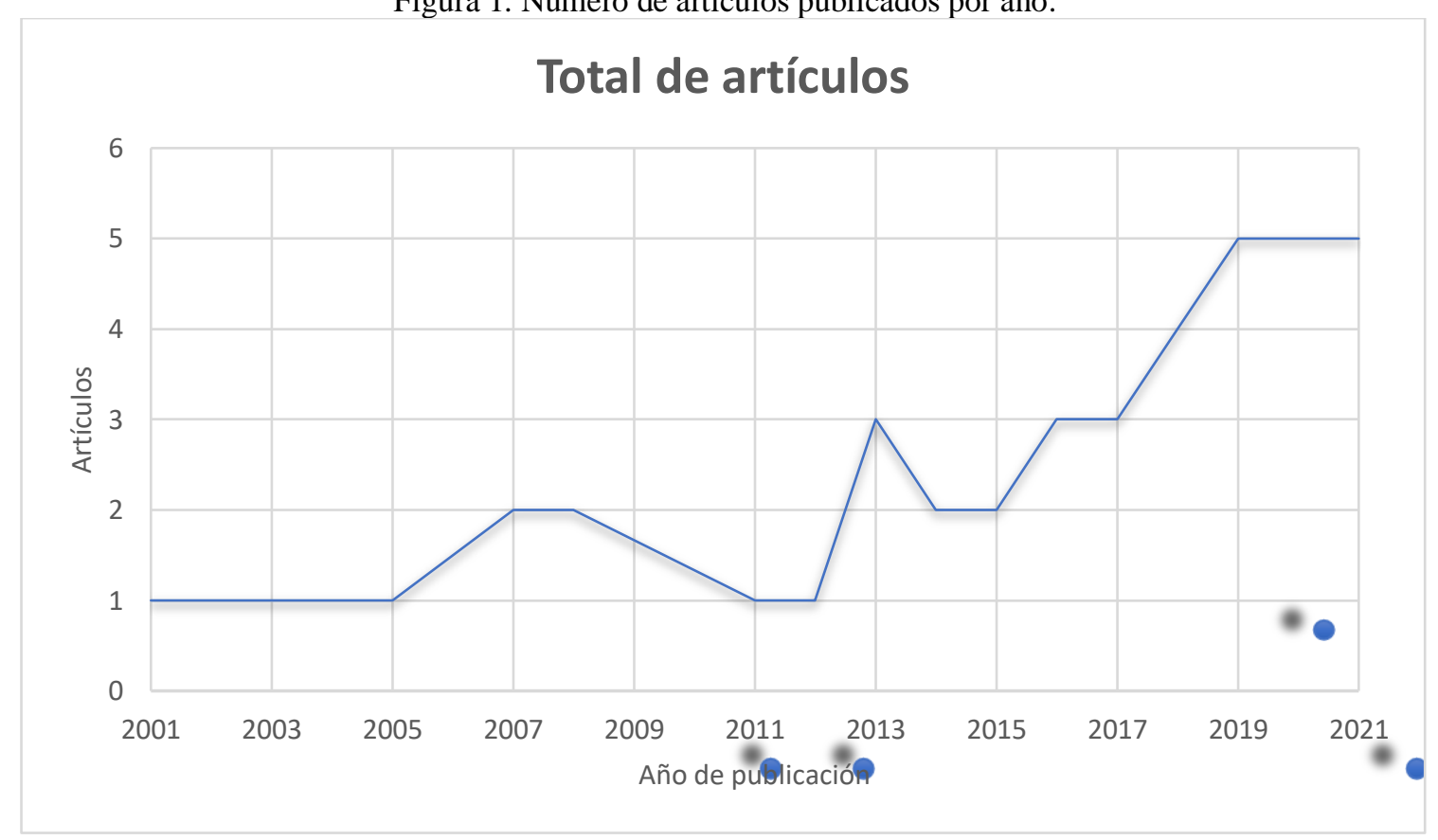

\section{1 ÍNDICE DE PRICE}

Trata de la proporción del número de referencias menor a cinco años de antigüedad, respecto al total de referencias.

$$
\begin{aligned}
& \text { IP 2001-2004 = (Documentos- } 5 \text { años x 100\%) /Total } \\
& \text { [Ec. 1] } \\
& \text { IP } 2001-2004=(1-5 \text { años } x 100 \%) / 40 \\
& \text { IP } 2001-2004=0,1 \\
& \text { IP } 2005-2009=(\text { Documentos- } 5 \text { años x 100\%) } / \text { Total } \\
& \text { [Ec. 2] } \\
& \text { IP } 2005-2009=(5-5 \text { años } x \text { 100\% }) / 40 \\
& \text { IP } 2001-2009=0
\end{aligned}
$$




$$
\begin{aligned}
& \text { IP 2010-2014 = (Documentos- } 5 \text { años x 100\%) /Total } \\
& \text { IP } 2010-2014=(7-5 \text { años } x 100 \%) / 40 \\
& \text { IP } 2010-2014=0,05 \\
& \text { IP 2015-2019 = (Documentos- } 5 \text { años x 100\%)/Total } \\
& \text { [Ec. 4] } \\
& \begin{aligned}
\text { IP } 2015-2019= & (\text { Documentos- } 5 \text { años x 100\% }) / \text { Total } \\
& \text { IP 2015-2019 }=(17-5 \text { años x 100\% }) / 40
\end{aligned} \\
& \text { IP } 2015-2019=0,3 \\
& \text { IP 2020-2021 = (Documentos- 3años x 100\%)/Total } \\
& \text { [Ec. 5] } \\
& \text { IP } 2020-2021=(10-3 \text { años x 100\% }) / 40 \\
& \text { IP } 2020-2021=0,175
\end{aligned}
$$

Al aplicar el índice de Price donde obtuvo un valor de 0,1 para los años 2001 a 2004, un valor de 0 para los años de 2005 a 2009, un valor de 0,05 para los años 2010 a 2014, un valor de 0,3 para los años de 2015 a 2019 y un valor de 0,175 para los años de 2020 a 2021, donde estos dos últimos índices de Price son los más altos, debido a que se observó un incremento muy alto de las publicaciones sobre los temas relacionados a la búsqueda de investigación.

\section{2 ÍNDICE DE COLABORACIÓN}

Para el año 2001, se registró el valor de un artículo escrito por 15 autores, con este valor se obtuvo un índice de colaboración de 15.

$$
\begin{gathered}
\frac{\sum_{\iota=1}^{n} J \iota n \iota}{N} \quad[E c .6] \\
\text { IC 2001= } \frac{(1 * 15)}{1}=15
\end{gathered}
$$

Para el año 2005, se registró el valor de un artículo escrito por 5 autores, con este valor se obtuvo un índice de colaboración de 5.

$$
\frac{\sum_{\iota=1}^{n} J_{\iota n \iota}}{\mathrm{N}}
$$




$$
\text { IC } 2005=\frac{(1 * 5)}{1}=5
$$

Para el año 2007, se registró el valor de un artículo escrito por 3 autores, otro artículo escrito por 2 autores, con estos valores se obtuvo un índice de colaboración de 2,5.

$$
\begin{gathered}
\frac{\sum_{\iota=1}^{n} J \iota n \iota}{\mathrm{N}} \\
\text { IC } 2007=\frac{(1 * 3)+(1 * 2)}{2}=2,5
\end{gathered}
$$

Para el año 2008, se registró el valor de un artículo escrito por 2 autores, otro artículo escrito por 3 autores, con estos valores se obtuvo un índice de colaboración de 2,5.

$$
\begin{gathered}
\frac{\sum_{\iota=1}^{n} J \iota n \iota}{\mathrm{N}} \\
\text { IC } 2008=\frac{(1 * 2)+(1 * 3)}{2}=2,5
\end{gathered}
$$

Para el año 2011, se registró el valor de un artículo escrito por un autor, con este valor se obtuvo un índice de colaboración de 1.

$$
\begin{gathered}
\frac{\sum_{\iota=1}^{n} J \iota n \iota}{\mathrm{N}} \\
\text { IC } 2011=\frac{(1 * 1)}{1}=1
\end{gathered}
$$

Para el año 2012, se registró el valor de un artículo escrito por dos autores, con este valor se obtuvo un índice de colaboración de 2. 


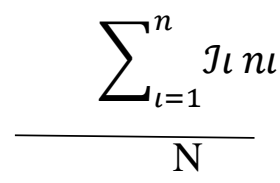

$$
\text { IC } 2012=\frac{(1 * 2)}{1}=2
$$

Para el año 2013, se registró el valor de un artículo escrito por 1 autor, otro artículo escrito por 6 autores, otro artículo escrito por 5 autores, con estos valores se obtuvo un índice de colaboración de 4.

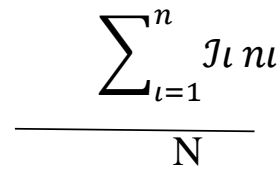

$$
\text { IC } 2013=\frac{(1 * 1)+(1 * 6)+(1 * 5)}{3}=4
$$

Para el año 2014, se registró el valor de un artículo escrito por un autor y otro artículo escrito por un autor, con estos valores se obtuvo un índice de colaboración de 1.

$$
\begin{gathered}
\frac{\sum_{\iota=1}^{n} J \iota n \iota}{\mathrm{N}} \\
\text { IC } 2014=\frac{(1 * 1)+(1 * 1)}{2}=1
\end{gathered}
$$

Para el año 2015, se registró un valor de un artículo escrito por 4 autores y otro artículo escrito por 3 autores, con estos valores se obtuvo un índice de colaboración de 3,5.

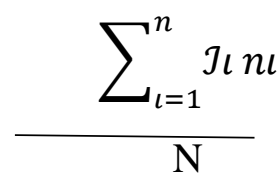




$$
\text { IC } 2015=\frac{(1 * 4)+(1 * 3)}{2}=3,5
$$

Para el año 2016, se registró un valor de un artículo escrito por un autor, otro artículo escrito por un autor, un último artículo escrito por 2 autores, con este valor se obtuvo un índice de colaboración de 1,7 .

$$
\begin{gathered}
\frac{\sum_{\iota=1}^{n} J \iota n \iota}{\mathrm{N}} \\
\operatorname{IC~} 2016=\frac{(1 * 1)+(1 * 1)+(1 * 3)}{3}=1,7
\end{gathered}
$$

Para el año 2017, se registró un valor de un artículo escrito por 8 autores, otro artículo escrito por 4 autores, otro artículo escrito por un autor, con este valor se obtuvo un índice de colaboración de 4,3.

$$
\begin{gathered}
\frac{\sum_{\iota=1}^{n} J \iota n \iota}{\mathrm{N}} \\
\operatorname{IC} 2017=\frac{(1 * 8)+(1 * 4)+(1 * 1)}{3}=4,3
\end{gathered}
$$

Para el año 2018, se registró un valor de un artículo escrito por 2 autores, otro artículo escrito por un autor, otro artículo escrito por 3 autores, un último artículo escrito por 7 autores, con este valor se obtuvo un índice de colaboración de 3,25.

$$
\begin{gathered}
\frac{\sum_{\iota=1}^{n} J \iota n \iota}{\mathrm{N}} \\
\operatorname{IC~} 2018=\frac{(1 * 2)+(1 * 1)+(1 * 3)+(1 * 7)}{4}=3,25
\end{gathered}
$$


Para el año 2019, se registró un valor de un artículo escrito por 3 autores, otro artículo escrito por 4 autores, otro artículo escrito por un autor, otro artículo escrito por un autor, otro artículo escrito por 2 autores, con este valor se obtuvo un índice de colaboración de 2,2.

$$
\begin{gathered}
\frac{\sum_{\iota=1}^{n} J \iota n \iota}{\mathrm{N}} \\
\operatorname{IC} 2019=\frac{(1 * 3)+(1 * 4)+(1 * 1)+(1 * 1)+(1 * 2)}{5}=2,2
\end{gathered}
$$

Para el año 2020, se registró un valor de un artículo escrito por 6 autores, otro artículo escrito por 4 autores, otro artículo escrito por 4 autores, otro artículo escrito por un autor, otro artículo escrito por 2 autores, con este valor se obtuvo un índice de colaboración de 3,4.

$$
\begin{gathered}
\frac{\sum_{\iota=1}^{n} J \iota n \iota}{\mathrm{N}} \\
\mathrm{IC} 2020=\frac{(1 * 6)+(1 * 4)+(1 * 4)+(1 * 1)+(1 * 2)}{5}=3,4
\end{gathered}
$$

Para el año 2021, se registró un valor de un artículo escrito por 6 autores, otro artículo escrito por 7 autores, otro artículo escrito por un autor, otro artículo escrito por un autor, otro artículo escrito por 2 autores, con este valor se obtuvo un índice de colaboración de 3,4.

$$
\begin{gathered}
\frac{\sum_{\iota=1}^{n} J \iota n \iota}{\mathrm{N}} \\
\operatorname{IC~} 2021=\frac{(1 * 6)+(1 * 7)+(1 * 1)+(1 * 1)+(1 * 2)}{5}=3,4
\end{gathered}
$$


De manera comparativa el índice de colaboración entre los años 2001 a 2021, presentó un valor de 15 para el año 2001, 5 para el año 2005, 2,5 para el año 2007, 2,5 para el año 2008, 1 para el año 2011, 2 para el año 2012, 4 para el año 2013, 1 para el año 2014, 3,5 para el año 2015, 1,7 para el año 2016, 4,3 para el año 2017, 3,25 para el año 2018, 2,2 para el año 2019, 3,4 para el año 2020, 3,4 para el año 2021.

Figura 2. Evolución del índice de colaboración período 2001-2021.

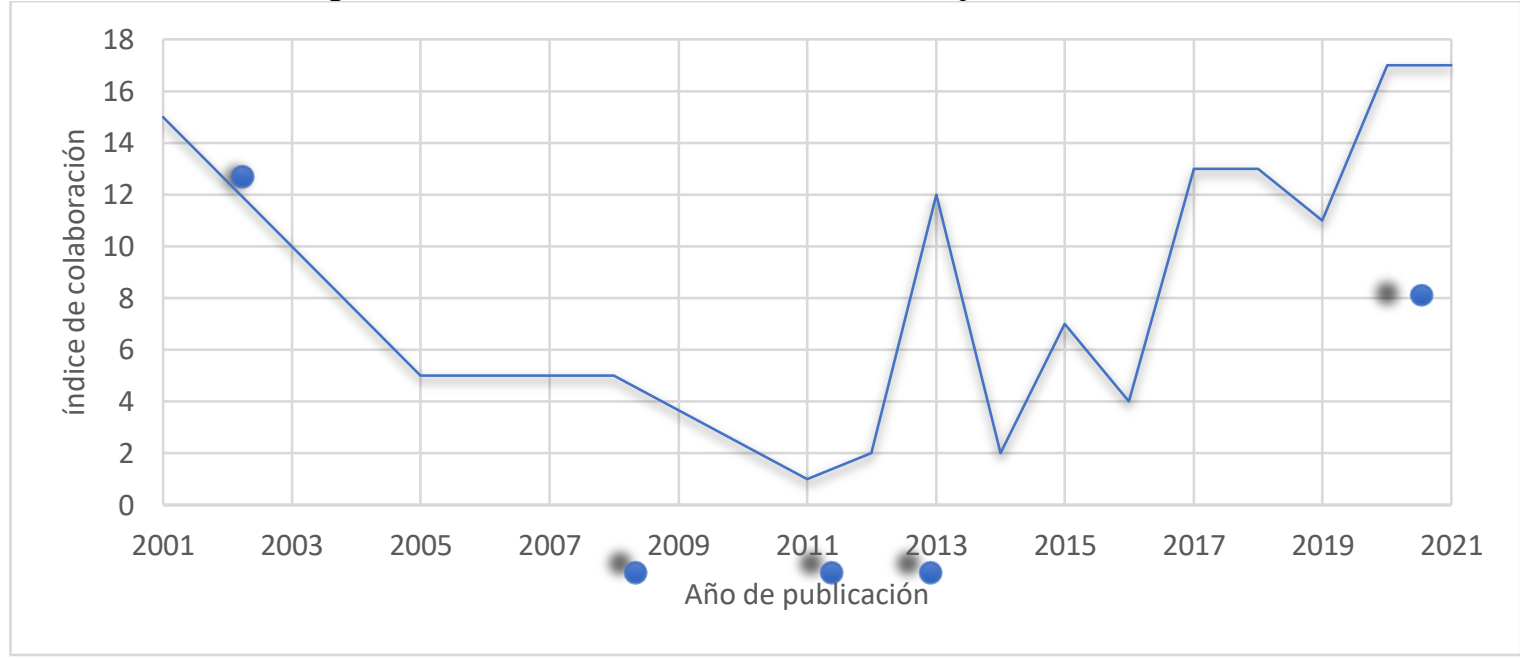

Figura 3. Total de referencias por año.

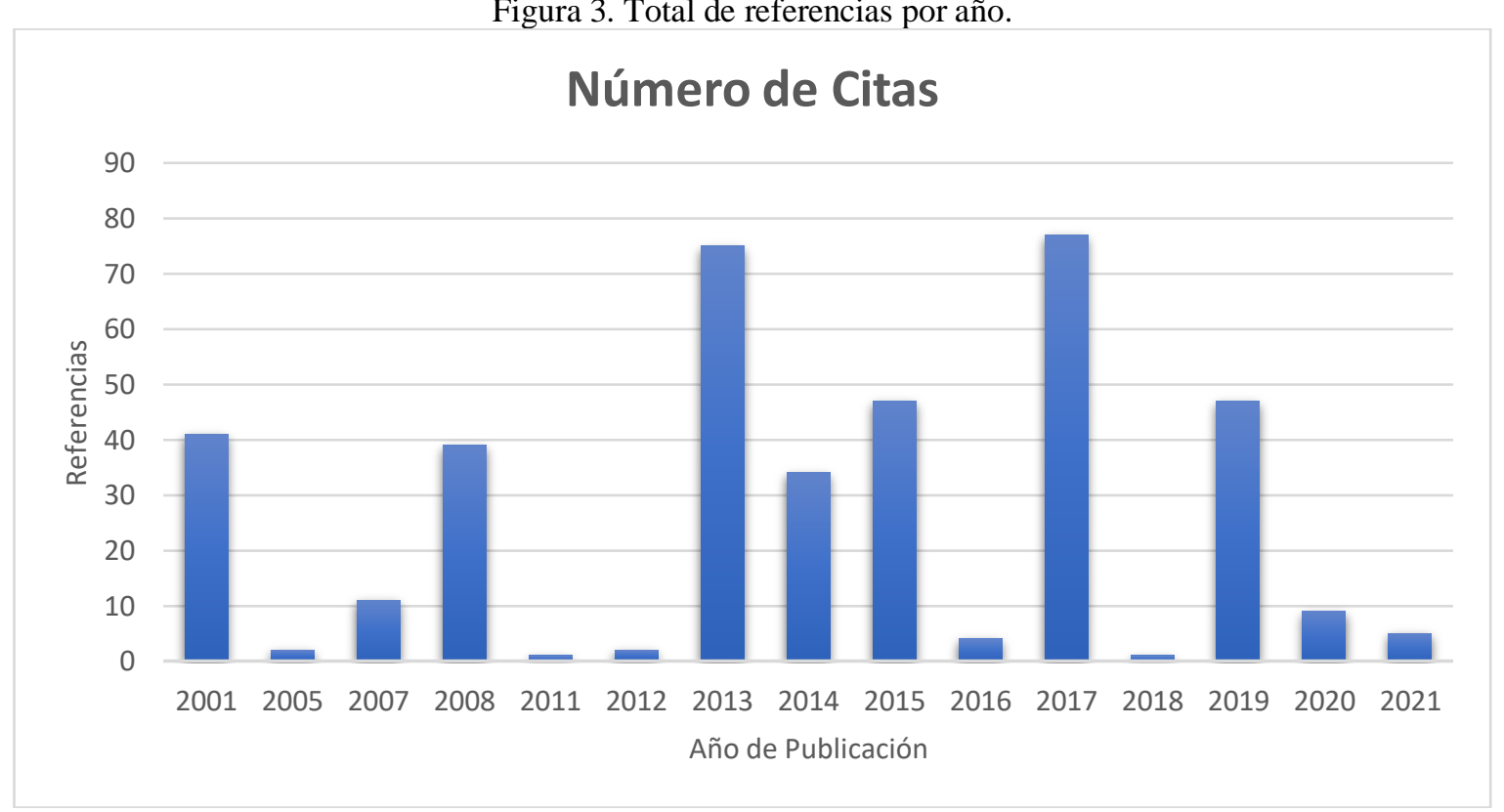


Figura 4. Total de autores por año.

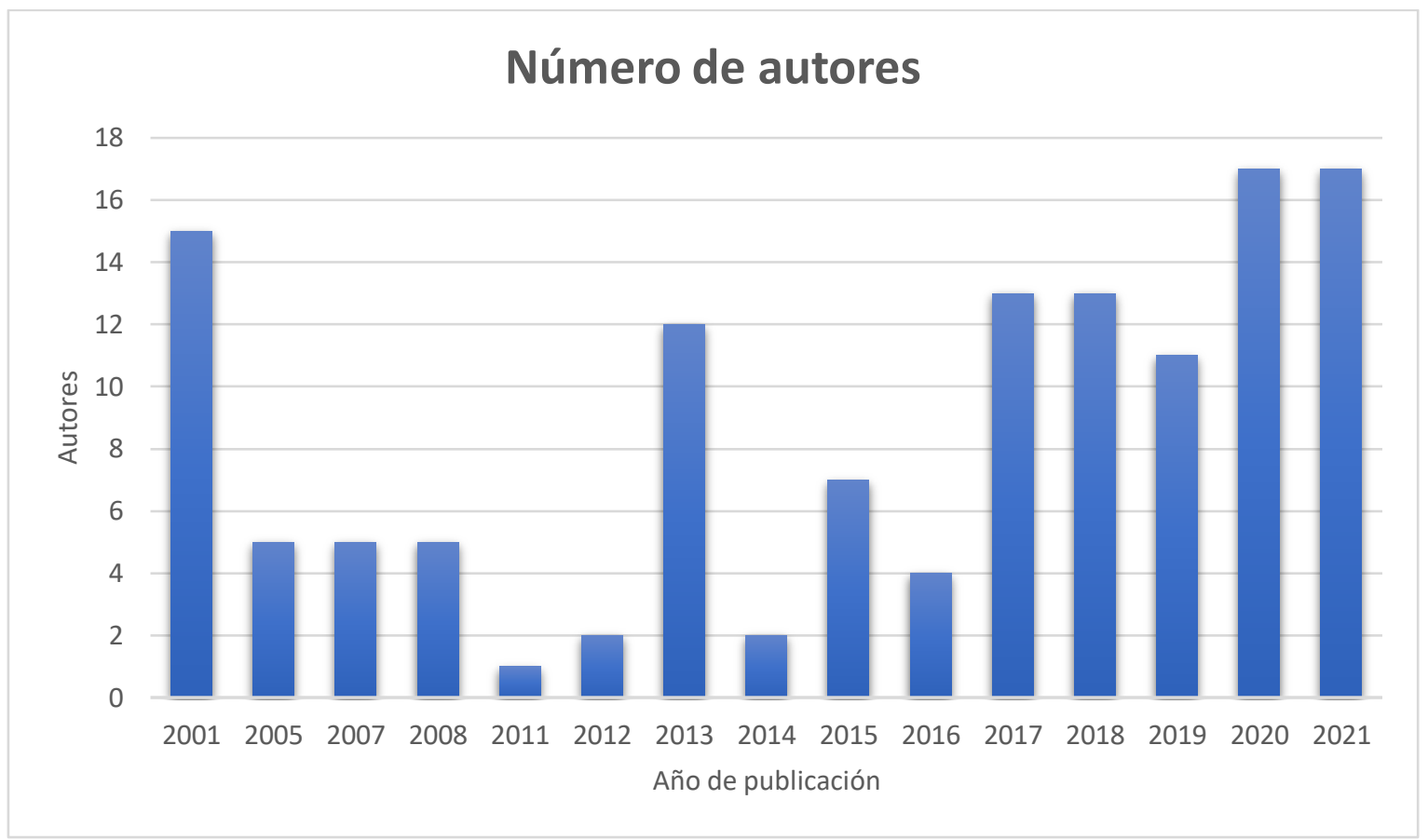

Cabe resaltar que, dentro de los 40 artículos, se destacan los últimos 3 años donde se puede observar que se encuentra la mayor cantidad de los artículos encontrado, con un total de 15, 61 citaciones y en los cuales intervinieron un total de 45 autores.

Luego de haber analizado las tendencias en la distribución de artículos por año (figura 1), las publicaciones de los últimos 3 años demuestran los valores más altos en cuanto a número de artículos encontrados. Esto indica que en los últimos años ha venido incrementando el interés de las personas por los temas relacionados con esta investigación.

Las palabras más importantes y por las cuales predominaban más en los títulos de los artículos siguen los objetivos de estudio en este análisis bibliométrico acerca del fenómenos de las heladas, los sistemas de riego y las estrategias que tienen los agricultores para mitigar estos daños. Las 3 palabras principales y fuentes frecuentes de información fueron: heladas, sistemas de riego, soluciones a las heladas.

El análisis también revelo las palabras clave de los autores que aparecieron en los artículos. Las palabras más comunes fueron, heladas, fenómeno de heladas, sistemas de riego, soluciones o estrategias a las heladas. De igual forma, con el respectivo análisis en cuanto al enfoque de los artículos la temática de las soluciones y/o estrategias que se utilizan hoy en día fue el tema de mayor interés con un rango de $45 \%$, luego sigue el de los sistemas de riego con un $30 \%$ y para finalizar tenemos el tema de las heladas y todo lo relacionado con este fenómeno con un $25 \%$. 
Se presenta una alta tasa de universidades a nivel nacional que tienen referencia alguna con los temas tratados en esta investigación que esta comprendida durante los periodos de 2001 a 2021. La mayoría de las universidades a nivel nacional no presento publicaciones en otro idioma, sin embargo, en la investigación se encontró información valiosa en 7 universidades, las cuales tuvieron un total de 12 publicaciones, lo cual indica que la temática estudiada tiene más interés a nivel mundial pero aun así el país esta empezando con un gran número de publicaciones que tienen interés sobre los diferentes temas relacionados con esta investigación.

En cuanto a revistas que publicaron más sobre el tema de las heladas, los sistemas de riego y estrategias y/o soluciones que tienen que ver con mitigar los daños causados por las heladas, se encontraron varias revistas a nivel mundial, como, por ejemplo, en Brasil, Ecuador, Estados Unidos entre otros países. De igual forma, las bases de datos como Science Direct, Scopus, Google Scholar y demás, tienen un papel importante debido a que en estas bases de datos se encontraron varios artículos relacionados a los temas de esta investigación que son de manera muy importante.

\begin{tabular}{|l|c|c|}
\hline \multicolumn{1}{|c|}{ PAÍS } & TOTAL ARTÍCULOS & TOTAL ARTÍCULOS (\%) \\
\hline Colombia & 14 & 35 \\
\hline Brasil & 6 & 15 \\
\hline Estados Unidos & 3 & 7,5 \\
\hline España & 2 & 5 \\
\hline China & 2 & 5 \\
\hline Iran & 2 & 5 \\
\hline
\end{tabular}

De acuerdo con el idioma de publicación, el 42,5\%, (17) artículos se encuentran en español, el $40 \%$ (16) se encuentran en ingles y el 17,5 (7) se encuentran en portugués 
Figura 5. Distribución de artículos por idioma de publicación

\section{Porcentaje artículos}

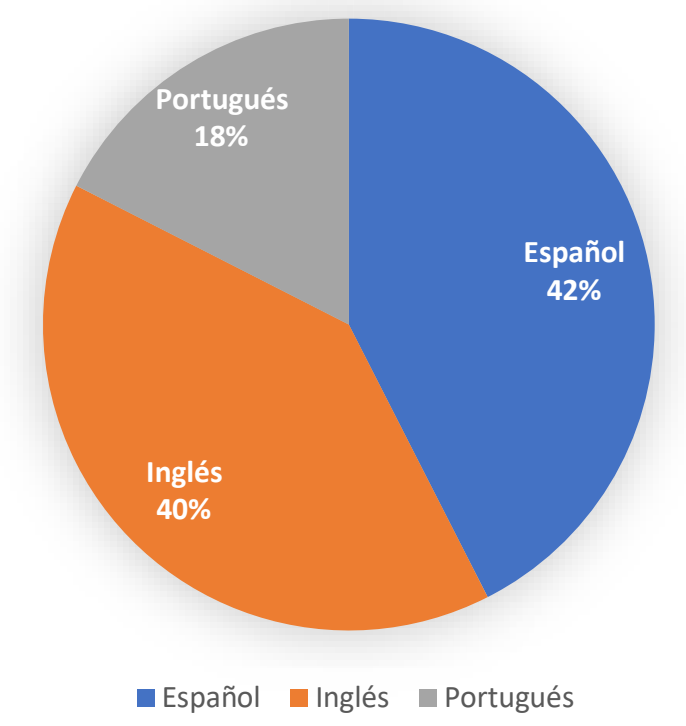

En cuanto a las temáticas que se abordan en la investigación sobre heladas, sistemas de riego y soluciones y/o estrategias para minimizar el daño causado por las heladas, se encontraron estos enfoques los cuales se pueden clasificar como ya se menciono anteriormente. Se estudian estos enfoques para conocer de mejor manera las actividades o referencias que en los artículos se puedan encontrar y sirva como orientación adecuada a la temática investigada y de igual manera el propósito que tiene importancia en la temática ambiental de la investigación.

El análisis bibliométrico de los artículos publicados sobre heladas, afectaciones de las heladas, sistemas de riego y soluciones y/o estrategias que se pueden encontrar hoy en día para minimizar el daño causado por el fenómeno de las heladas encontrados en las bases de datos como Science Direct, Scopus, de igual forma, en Google Scholar, permite evidenciar algunas características sobre la investigación que se desarrolla en algunos países del mundo sobre las estrategias que se realizan para mitigar el daño causado por las heladas y los sistemas de riego que se utilizan a nivel de la agricultura. La mayoría de estos artículos encontrados son de investigación y están encaminados hacia mejorar las condiciones de los agricultores a nivel mundial, donde se reconoce la incorporación de nuevas técnicas para ayudar a mitigar este daño causado por las heladas.

\section{CONCLUSIONES}

Se presentó un reseña de la investigación sobre el fenómeno de las heladas, afectaciones que tienen las heladas, sistemas de riego y soluciones y/o estrategias que se pueden encontrar hoy en día para 
minimizar el daño causado por este fenómeno durante el periodo comprendido entre 2001 a 2021 con respecto a los resultados de publicaciones anuales, las bases de datos como Science Direct, Scopus y Google Scholar, áreas de investigación, artículos, países. El resultado del número de publicaciones en estos temas presentó un incremento muy alto en los últimos años, esto quiere decir, que la investigación sobre el fenómeno de las heladas, afectaciones que tienen las heladas, sistemas de riego y soluciones y/o estrategias que se pueden encontrar hoy en día para minimizar el daño causado por este fenómeno es un campo de estudio muy amplio e importante hoy en día no solo a nivel nacional sino a nivel mundial también. Como la investigación se piensa implementar en Colombia, una parte de los artículos se encontró en el país, considerando a Colombia y Brasil como los países donde más artículos se encontraron referente a los temas relacionados con la investigación.

El incremento en la producción de artículos hacía la temática de la heladas, los sistemas de riego y soluciones y/o estrategias que se encuentran para mitigar este fenómeno, puede verificarse mediante los índices elaborados en la investigación, como lo es el índice de colaboración que presentó un valor alto de 15 para el año 2001, 5 para el año 2005, 4,3 para el año 2017, 3,4 para el año 2020, 3,4 para el año 2021, en comparación con otros datos como 1 para el año 2011, 1 para el año 2014, 1,7 para el año 2016, 2 para el año 2012, demostrando así que el tema de las heladas, sus afectaciones y las soluciones que se presentan hoy en día han venido teniendo un auge muy importante a nivel mundial, además de todo lo relacionado con el cambio climático y las variables climáticas que cada día aumentan aún más, tema que es de mucha importancia hoy en día y es por esto que se hace importante realizar investigaciones, consultas y demás cosas, con el fin de conocer mejor sobre el tema, asesorarse y ver a nivel no solo nacional sino mundial como estos temas tienen gran importante con el pasar de los días.

Teniendo en cuenta el área de investigación en la cual se realizó el análisis bibliométrico, se tiene que establecer criterios y parámetros muy claros para la selección de bases de datos, de igual forma las palabras claves y el enfoque que es importante a la hora de realizar este análisis. Es muy importante la participación educativa a nivel nacional y mundial con base al tema relacionado de esta investigación y como estos temas vienen tomando mucha fuerza y hoy en día tienen gran importancia. Cabe resaltar la importancia en cuanto a la producción de artículos relacionados con las heladas, sus afectaciones a nivel nacional, los sistemas de riego y soluciones y/o estrategias que se encuentran para mitigar este fenómeno, en Colombia, debido a que es el país donde más artículos relacionado con este tema se encontraron. 


\section{REFERENCIAS}

Altieri, M. A., \& Nicholls, C. (2008). Los impactos del cambio climático sobre las comunidades campesinas y de agricultores tradicionales y sus respuestas adaptativas. Agroecología, 3, 7-24.

Alvarado Gaona, A. E., \& Pérez Fagua, C. (2007). Caracterización de zonas sensibles a heladas en el cultivo de papa (solanum tuberosum) en boyacá. Caracterización De Zonas Sensibles a Heladas En El Cultivo De Papa (Solanum Tuberosum) En Boyacá,

BENYEZZA, H., BOUHEDDA, M., \& REBOUH, S. (2021). Zoning irrigation smart system based on fuzzy control technology and IoT for water and energy saving. Journal of Cleaner Production, , 127001. doi:https://doi-org.crai-ustadigital.usantotomas.edu.co/10.1016/j.jclepro.2021.127001

Cadenas, J. M., Garrido, M. C., Martínez-España, R., \& Guillén-Navarro, M. A. (2020). Making decisions for frost prediction in agricultural crops in a soft computing framework. Computers and Electronics in Agriculture, 175, 105587. doi:https://doi-org.craiustadigital.usantotomas.edu.co/10.1016/j.compag.2020.105587

Cisneros, F., Pacheco T, E., \& Feyen, J. (2007). Evaluación del rendimiento de sistemas de riego por aspersión de baja pluviosidad como resultado de la aplicación de la extensión como soporte técnico. Ingeniería Del Agua, 14(3), 177-185.

da Cunha, G. R., Haas, J. C., Maluf, J. R. T., Henrique, P., Gonçalves, S., Wrege10, M., .. Pimentel13, M. B. M. (2001). Zoneamento agrícola e época de semeadura para trigo no brasil. Revista Brasileira De Agrometeorologia, Passo Fundo, 9(3), 400-414.

de Oliveira Paulino, Marney Aparecida, de Figueiredo, F. P., Fernandes, R. C., Maia, Janini Tatiane Lima Souza, de Oliveira Guilherme, D., \& Barbosa, F. S. (2013). Avaliação da uniformidade e eficiência de aplicação de água em sistemas de irrigação por aspersão convencional. Revista Brasileira De Agricultura Irrigada-Rbai, 3(2)

de Souza, L. O., Mantovani, E. C., Soares, A. A., Ramos, M. M., \& de Freitas, P. S. (2006). Avaliação de sistemas de irrigação por gotejamento, utilizados na cafeicultura. Revista Brasileira De Engenharia Agrícola E Ambiental, 10, 541-548.

Demin, P. (2014). Aportes para el mejoramiento del manejo de los sistemas de riego. Métodos De Riego: Fundamentos, Usos Y Adaptaciones.Ediciones INTA, San Fernando Del Valle De Catamarca,

Díaz Arévalo, J. L.Aprovechamiento de aguas superficiales para mini-distrito de riego en la vereda "Labrancitas", en paz de ariporo, casanare.

Estacio Rodriguez, C. A., \& Ariza Garzón, A. M. (2020). Sistema de protección ante las heladas, para los cultivos de papa del territorio cundiboyacense. AMKHA.

Feng, X., Yan, F., \& Liu, X. (2019). Study of wireless communication technologies on internet of things for precision agriculture. Wireless Personal Communications, 108(3), 1785-1802. doi:10.1007/s11277019-06496-7 
Firfiris, V. K., Fragos, V. P., Kotsopoulos, T. A., \& Nikita-Martzopoulou, C. (2020). Energy and environmental analysis of an innovative greenhouse structure towards frost prevention and heating needs conservation. Sustainable Energy Technologies and Assessments, 40, 100750. doi:https://doi-org.craiustadigital.usantotomas.edu.co/10.1016/j.seta.2020.100750

Fonseca Forero, L. C., \& Lancheros Coy, D. A.Evaluación regional de escenarios de cambio climático en boyacá.

Goméz Ramírez, D. C. (2019). Diagnóstico en terrenos inclinados para el desarrollo de distritos de riego, caso de estudio: Veredas de la zona norte del municipio de paipa.

Gomez Ramirez, J. A., \& Molina Ortiz, J. F. (2012). Sistema para la disminucion de daños causados por las heladas. Sistema Para La Disminucion De Daños Causados Por Las Heladas,

González Botero, D. F. (2018). Planteamiento de un modelo de predicción de heladas en cultivos de rosa en la sabana de bogotá.

Guillén-Navarro, M. A., Martínez-España, R., Bueno-Crespo, A., Morales-García, J., Ayuso, B., \& Cecilia, J. M. (2020). A decision support system for water optimization in anti-frost techniques by sprinklers. Sensors (Switzerland), 20(24), 1-15. doi:10.3390/s20247129

Gutierrez Gonzalez, D. S., \& Osorio Alarcón, L. H.Modelo de sequía en la quebrada aguas claras en el municipio de san bernardo cundinamarca a través del programa hidrológico SWAT mediante aplicación de herramientas digitales UAVs. Retrieved from https://repository.usta.edu.co/handle/11634/16598

Jaradat, M. A. K., Al-Nimr, M. A., \& Alhamad, M. N. (2008). Smoke modified environment for crop frost protection: A fuzzy logic approach. Computers and Electronics in Agriculture, 64(2), 104-110. doi:https://doi-org.crai-ustadigital.usantotomas.edu.co/10.1016/j.compag.2008.04.007

Lefort Valenzuela, Inti Hernán Manual. (2014). Propuesta de un indicador para generar información válida y oportuna sobre la ocurrencia de heladas en la región de coquimbo.

Leguizamón Martínez, J. S. (2016). Sistema electrónico de protección contra las heladas que afectan los cultivos de papa.

Mohammadi, H., Moghbel, M., \& Ranjbar, F. (2016). Prediction of soil frost penetration depth in northwest of iran using air freezing indices. Theoretical \& Applied Climatology, 126(3-4), 533-541. doi:10.1007/s00704-015-1564-1

Narváez López, D. E.Revisión del funcionamiento de un distrito de riego a pequeña escala en el municipio de potosi-nariño, empleando software de simulación.

Noh, I., Doh, H. -., Kim, S. -., Kim, S. -., Shin, S., \& Lee, S. -. (2021). Machine learning-based hourly frost-prediction system optimized for orchards using automatic weather station and digital camera image data. Atmosphere, 12(7) doi:10.3390/atmos12070846

Oliveira, Guilherme da Silva de. (2011). Avaliação da fenologia de cinco cultivares de mirtilo (vaccinium sp.) no município de bom retiro (SC) e as implicações na suscetibilidade a geadas e no manejo dessas cultivares. 
Olszewski, F., Jeranyama, P., Kennedy, C. D., \& DeMoranville, C. J. (2017). Automated cycled sprinkler irrigation for spring frost protection of cranberries. Agricultural Water Management, 189, 1926. doi:https://doi-org.crai-ustadigital.usantotomas.edu.co/10.1016/j.agwat.2017.04.014

Pazouki, E. (2021). A practical surface irrigation system design based on volume balance model and multi-objective evolutionary optimization algorithms. Agricultural Water Management, 248, 106755. doi:https://doi-org.crai-ustadigital.usantotomas.edu.co/10.1016/j.agwat.2021.106755

Pradilla Villamizar, G. (2016). Análisis ambiental de las prácticas campesinas de resiliencia a la variabilidad y el cambio climático en fincas ecológicas del altiplano cundiboyacense-colombia. Instituto De Estudios Ambientales (IDEA),

Priya, P., \& Madhumitha, R. (2021). Modelling and simulation for automatic irrigation system with PV solar tracking. Materials Today: Proceedings, doi:https://doi-org.craiustadigital.usantotomas.edu.co/10.1016/j.matpr.2021.03.146

Pulatov, B., Linderson, M., Hall, K., \& Jönsson, A. M. (2015). Modeling climate change impact on potato crop phenology, and risk of frost damage and heat stress in northern europe. Agricultural and Forest Meteorology, 214-215, 281-292. doi:https://doi-org.craiustadigital.usantotomas.edu.co/10.1016/j.agrformet.2015.08.266

Ros, C. O. D., Perrando, E. R., Paula, G. M. d., Somavilla, L., Predige, Daylien

Mayane Sossmeier Albring, Engel, K., \& Silva, R. F. d. (2018). RESISTÊNCIA À GEADA E CRESCIMENTO INICIAL DE toona ciliata EM CULTIVOS CONSORCIADOS COM eucalyptus grandis EM DIFERENTES ADUBAÇÕES. Ciência Florestal, 28, 796-808.

Rudy Hendrawan, I. N., Putu Yulyantari, L., Pradiptha, G. A., \& Bayu Starriawan, P. (2019). Fuzzy based internet of things irrigation system. Paper presented at the 2019 1st International Conference on Cybernetics and Intelligent System, ICORIS 2019; 1st International Conference on Cybernetics and Intelligent System, ICORIS 2019, 146-150. doi:10.1109/ICORIS.2019.8874900 Retrieved from https://www.scopus.com/inward/record.uri?eid=2-s2.0-

85074421058\&doi=10.1109\%2fICORIS.2019.8874900\&partnerID=40\&md5=99155192ba04e593214 $9 \mathrm{bbb} 40722 \mathrm{efab}$

Salamanca-Figueroa, F. A., Suarez-Rodrígez, W. A., \& Cárdenas-Gamboa, C. C. (2018). Prototipo de monitoreo y alarma para la detección de heladas blancas en sectores rurales de sogamoso. Ingeniería Investigación Y Desarrollo, 18(1), 57-64.

Santos, I. R. N. (2019). Desenvolvimento de uma aplicação informática para aferir a viabilidade técnica de métodos de luta contra geadas em fruticultura. Desenvolvimento De Uma Aplicação Informática Para Aferir a Viabilidade Técnica De Métodos De Luta Contra Geadas Em Fruticultura,

Torres Moya, E.Caracterización de las temperaturas extremas del aire para el cultivo de papa en la sabana de bogotá.

Urbano-Molano, F. A. (2013). Redes de sensores inalámbricos aplicadas a optimización en agricultura de precisión para cultivos de café en colombia. Journal De Ciencia E Ingenieria, 5(1), 46-52. 
Wang, P., Ma, Y., Tang, J., Wu, D., Chen, H., Jin, Z., \& Huo, Z. (2021). Spring frost damage to tea plants can be identified with daily minimum air temperatures estimated by modis land surface temperature products. Remote Sensing, 13(6) doi:10.3390/rs13061177

Wolfe, D. W., Peck, G. M., DeGaetano, A. T., Carey, M., Ziska, L. H., Lea-Cox, J., . . Hollinger, D. Y. (2018). Unique challenges and opportunities for northeastern US crop production in a changing climate. Climatic Change, 146(1), 231-245. doi:10.1007/s10584-017-2109-7

Zaro, G. C., Ricce, W. d. S., Caramori, P. H., Carvalho, S. L. C., \& Vicentini, M. E. (2014). Zoneamento agroclimático para a cultura do abacateiro no estado do paraná. Revista Brasileira De Fruticultura, 36, 363-372. 